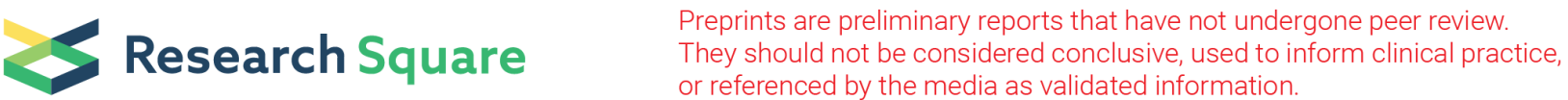 \\ Self-Made Distal Femoral Extramedullary Positioning Osteotomy Template In Total Knee Arthroplasty
}

\section{Sun zhe}

first affiliated hospital of dali university

\section{Dahir hussein}

Kunming Medical University second Affilliated Hospital https://orcid.org/0000-0001-8014-1513

\section{Zhao Gang}

kunming medical university second affiliated hospital

\section{Sun Chaojun (D13908725590@139.com )}

kunming medical university second affiliated hospital https://orcid.org/0000-0001-8441-6799

\section{Tariq Siraj Qazi}

kunming medical university second affiliated hospital

\section{yang kang}

kunming medical university second affiliated hospital

\section{Hu Zhifu}

first affiliated hospital of dali university

\section{Md Ariful Haque}

Yan an hospital affiliated to kunming medical university

\section{li Hong}

first affiliated hospital of dali university

\section{Zhao chang}

first affiliated hospital of dali university

\section{xu Jing}

first affiliated hospital of dali university

\section{Li Yue}

first affiliated hospital of dali university

\section{Sun Li}

Dali first peaple's hospital

\section{Research article}

Keywords: Total knee arthroplasty, Extramedullary distal femoral osteotomy template, Intramedullary positioning, knee replacement, positioning 
Posted Date: September 7th, 2021

DOl: https://doi.org/10.21203/rs.3.rs-850385/v1

License: (c) (i) This work is licensed under a Creative Commons Attribution 4.0 International License. Read Full License 


\section{Abstract}

Introduction: The objective of this study was to assess the success of total knee arthroplasty with the use of extramedullary positioning osteotomy templates for limb alignment in contrast to traditional intramedullary positioning rods.

Methods: Between September 2019 and February 2021, 70 participants underwent total knee arthroplasty with lower limb positioning using an extramedullary distal femur positioning template and intramedullary positioning rods. The participants were divided into two groups each to receive either of the two limb positioning techniques. The groups were divided randomly and were similar at the beginning of the study. Therefore, the participants were equally divided into 35 participants per group. Participants' indication for the procedure was severe osteomyelitis or rheumatoid arthritis, and they underwent distal femoral osteotomy. The force lines of the lower limb were identified by locating the center of the head of the femur.

The patients were monitored for intraoperative bleeding, surgical trauma and surgical time. They were followed up during the first and second weeks post-operation to assess for knee joint movement graded using the HSS knee score and assessed for deviation. Participants were treated equally throughout the study and during the follow-up phase.

Results: The participants did not have any statistically significant differences at the beginning of the study. The intramedullary group had a lesser average age but without any significant difference from the extramedullary group. In addition, their HSS knee scores, body mass index, knee joint range of motion, and the angle of deviation of the knee, hip, and ankle were of no statistical significance with a p-value of more than 0.05 .

The operation time for the extramedullary group was approximately 96.57 minutes with a margin of error of (+/-) 8.576 minutes. In contrast, the intramedullary group had an average time of 104.8 minutes and a margin of error of (+/-) 8.864. Intraoperative bleeding for the extramedullary group was less at approximately $89.97 \mathrm{ml}$, while it was $95.4 \mathrm{ml}$ in the intramedullary group. Postoperative drainage was also more in the intramedullary group as compared to the intramedullary group. The differences between both groups were statistically significant, with a $p$-value of less than 0.05 . There was no significant difference in HKA angle deviation, coronal plane angle, and flexion angle of femoral prosthesis $(P>0.05)$.

Conclusion: Extramedullary distal femoral osteotomy positioning templates proved superior to intramedullary positioning rods. The template demonstrated less surgical trauma hence the lower risk of deformity, less intraoperative bleeding, therefore, more safety and less risk for infection than the intramedullary positioning rods.

\section{Introduction}


For the longest time, the alignment of the limb following total knee arthroplasty has been one of the defining factors for the procedure's success. The parameters to measure the success of arthroplasty are both acute and chronic. The acute factors are those influencers that happen in the immediate period of the procedure. In the case of a surgical operation, that implies intraoperative factors. Chronic influencers are those that occur in the healing period following the operation. Among the operative risks that are uniform for all surgical procedures are intraoperative hemorrhage and traumatic events during operation. Traumatic events imply both predicted and unpredicted surgical trauma. Therefore, limb alignment has been an integral factor of the correct healing of limbs that have undergone total knee arthroplasty. The necessity to achieve the correct alignment is, therefore, driven by an intention to treat.

In patients who have undergone total knee arthroplasty, the limb is delicate with reduced tone. The nature of the postoperative conditions means that the limb cannot maintain alignment or position without extra support. For that purpose, doctors resulted utilizing medullary aligning tools to achieve the desired results [1]. For the longest time, intramedullary positioning rods have been the primary means to achieve alignment. The intramedullary positioning rods entail placing the positioning rods within the femur to achieve the correct alignment [1]. The procedure is long, tedious, and has many appendage risks. Advancements in technology have attempted to improve the outcomes and the procedure, but that has achieved little progress.

Intramedullary positioning rod placement is a long procedure that necessitates lengthy preoperative planning. The angle of deviation calculated through finding a center from three points of the ankle, knee, and hip joint allows for the planning to achieve some degree of accuracy [2] However, to achieve such precision, the planning process often involves prolonged and consecutive imaging. One way to improve precision in these procedures is to utilize imaging-guided computer-assisted measures [3]. However, since computers improve their accuracy by studying images, the patient is often exposed to radiation [2]. Computer systems utilize 3-D printing technology to improve their accuracy by measuring the lower extremity force lines [4]. While the approach has improved the accuracy of the procedure, there has been the challenge of prolonged exposure to $x$-ray radiation [3] The computers also take time to study the images and further prolong the preoperative preparation that could worsen the patients' condition.

Another demerit associated with the procedure is the marrow expansion and risk of infection. Abnormal marrow expansion can result in further skeletal degradation as bones reduce in density. Marrow suppression may also result in abnormal blood cells. Most blood cells are produced in the bone marrow, including leukocytes involved in immunity. Therefore, compromise on the bone marrow results in reduced immunity as it interferes with white blood cell production. The placement of the intramedullary nails within the medulla also increases the risk of infection directly from the positioning rods that could spread throughout the body through the lymphatic or hematogenous spread.

Intramedullary positioning rods have also been associated with intraoperative risks. The most common intraoperative risks are intraoperative hemorrhage and surgical trauma. The procedure is lengthy due to the caution required to achieve the desired positioning and, consequently, alignment. In addition, the rods 
require to be hoisted on the bone using screws hence necessitates drilling into bone tissue. The intricacy of the procedure and the prolonged length increases the risk of intraoperative bleeding. Bleeding can easily complicate and result in loss of life, and it is an integral parameter to account for while assessing for the safety of any procedure.

The second intraoperative risk is surgical trauma. As already mentioned, intramedullary positioning rods require an invasion of the medulla of the femur and are therefore highly invasive. Total knee arthroplasty already involves a relatively high degree of surgical trauma as it involves severing the distal aspect of the femur. Surgical trauma increases healing periods and could result in healing with deformity due to loss of tissue. In addition, surgical trauma is an important aspect of procedural success because it determines its quality of life following the procedure. Therefore, the increased surgical trauma with intramedullary rods can be credited with high complication rates for patients following the procedure.

The increased risks and other disadvantages of intramedullary positioning rods have been reduced with extramedullary positioning osteotomy templates. Extramedullary templates reduce nearly all the risks associated with intramedullary rods and also act as an alternative for patients with contraindications from using intramedullary rods. For example, patients with deformed femoral stems or healing femoral stem fractures are contraindicated using intramedullary positioning rods. However, the intramedullary positioning rods also increase the risk of femoral fractures. On the other hand, extramedullary templates reduce the need for prolonged preoperative planning since they do not involve invasion of the medulla. Therefore, extramedullary templates do not involve extra costs, as would be when computer-guided and 3-D printing was used to increase accuracy for patients [4]. The reduced preoperative planning time also eliminates the need for prolonged exposure to $x$-ray radiation. Here fore, they are safer to that end.

Extramedullary templates demonstrate reduced intraoperative and postoperative risks. The risk of infection is significantly reduced with the templates than intramedullary rods since they are not as invasive and avoid marrow expansion. Less invasion means fewer blood vessels are injured, which translates to reduced risks of intraoperative bleeding. The procedure also involves significantly less surgical trauma, reducing the risk of patients healing with deformities. This study designed and developed an extramedullary femoral positioning osteotomy to be used for distal femur osteotomy. The study involved 70 cases of rheumatoid arthritis as well as osteomyelitis in advanced stages. The 70 participants were divided between the intramedullary and extramedullary groups to treat the approach designed to be a non-inferiority study. The study was conducted from September 2019 to February 2021. All parties were equal at the beginning of the trial, and they were treated equally throughout the study.

\section{Objective}

1. To assess the success of Total knee arthroplasty with the use of extramedullary positioning osteotomy templates for limb alignment.

\section{Materials And Methods}




\subsection{Research Methodology \\ 3.1.1 Objective of Study}

The study analyzed 70 cases conducted between September 2019 to February 2021. The group was divided into two groups of 35 individuals of both male and females. The inclusion criteria were patients who had severe osteoarthritis and rheumatoid arthritis. One group was placed to receive the traditional intramedullary positioning rod, while the second group received extramedullary distal femur templates. The groups were compared using follow-up data collected during the procedure focusing on intraoperative hemorrhage, surgical trauma, post one week and fortnight knee mobility, and X-ray imaging done to assess deviation. The deviation of interest was the angle between the hip, the ankle, and the knee

\subsection{General information:}

Both groups were similar at the beginning of the trial on all parameters. The intramedullary group had a lesser average age but without any significant difference from the extramedullary group. Their HSS knee scores, body mass index, knee joint range of motion, and the angle of deviation of the knee, hip, and ankle were of no statistical significance with a p-value of more than 0.05 . The defining parameters for their similarity have been

Table 1

Comparison of general preoperative data between extramedullary localization group and intramedullary

localization group ( $x \pm \mathrm{s}, 35$ cases)

\begin{tabular}{|c|c|c|c|c|c|}
\hline groups & $\begin{array}{l}\quad- \\
\text { age ( } x \\
\pm \text { s,years) }\end{array}$ & $\begin{array}{l}- \\
\text { BMI }(x \\
\left. \pm \mathrm{s}, \mathrm{kg} / \mathrm{m}^{2}\right)\end{array}$ & $\begin{array}{c}\text { HSS knee } \\
- \\
\text { score( } x \\
\pm \mathrm{s}, \mathrm{score})\end{array}$ & $\begin{array}{l}\text { Range of motion } \\
\text { of knee joint }\left(x \pm s^{\circ}{ }^{\circ}\right)\end{array}$ & $\begin{array}{l}\text { HKA angle } \\
\text { deviation }(x \\
\left. \pm s^{\circ}\right)\end{array}$ \\
\hline $\begin{array}{l}\text { Extramedullary } \\
\text { localization group }\end{array}$ & $\begin{array}{l}69.09 \pm \\
5.7\end{array}$ & $\begin{array}{l}23.611 \pm \\
2.73\end{array}$ & $48.77 \pm 5.48$ & $85.06 \pm 11.45$ & $10.486 \pm 3.32$ \\
\hline $\begin{array}{l}\text { Intramedullary } \\
\text { localization group }\end{array}$ & $\begin{array}{l}67.17 \pm \\
5.38\end{array}$ & $\begin{array}{l}24.617 \pm \\
3.0829\end{array}$ & $49.2 \pm 5.95$ & $85.77 \pm 11.44$ & $10.374 \pm 3.31$ \\
\hline$t$ & 1.445 & -1.444 & -0.314 & -0.261 & 0.141 \\
\hline$P$ & 0.153 & 0.153 & 0.755 & 0.795 & 0.889 \\
\hline
\end{tabular}

\subsection{Exclusion Criteria}

The exclusion criteria were with people who had a previous preoperative knee injury involving local ligaments. Patients with a history of knee operation would also give inaccurate findings that could influence the accuracy of the data collected. People with a history of gastric ulcers and allergies to NSAIDs as well as renal and hepatic impairment. Those groups were left out due to the need to use 
NSAIDs to manage pre-and post-operation patients. Contraindication to NSAIDs restricted participation. Patients who had a history of hemorrhagic disorders, specifically blood clotting disorders, were also excluded from the likelihood of their conditions interfering with the study [5]. The exclusion criteria were designed to reduce interference with the conditions.

\subsection{General information of the operation}

All patients were operated on by the same surgeons with intramedullary positioning rods and extramedullary femoral osteotomy templates from the same manufacturers based in three different countries. The goal was to have similar participants across both groups and ensure they were treated equally. Most of the patients received the Chinese-built GKPS with 16 cases in the extramedullary group and 15 cases in the intramedullary group. Eleven people received the German-made GEMINI prosthesis in both groups, while the American-made PFC PS was placed in 8 cases and 9 cases in the extramedullary and intramedullary groups

Respectively

\section{Surgical Methods}

The HKA angle of deviation necessitates finding the center for each joint. The center of the hip joint can be found by finding the center of the head of the femur [6].In this case, the C-arm x-ray was used to find the center of the head of the femur. Incision into the knee is median with consequent release of the bilateral tissues and excision of the cruciate ligaments both anteriorly and posteriorly osteophytes [7]. Extramedullary positioning was achieved, and the tibial facet was attached to the osteotomy with the knee in an extended position. Positioning with an $8-10 \mathrm{~mm}$ and $2.4 \mathrm{~mm}$ thickness on the lateral and medial aspects of the tibia [8]. A $0^{0}-30^{0}$ angle of retroversion control was achieved following the positioning.

Two spreader clamps were placed in the joint spaces laterally and medially between the proximal tibia and the distal femur. The clamps were intended to achieve ligament tension, and two clamps were used because of the medial and lateral ligaments [9].The internal and external distraction forceps to the internal and external ligaments to tension the lower limb force line.

\section{Clinical Assessment}

The factors used to determine the procedure's success were determined by the risks associated with intramedullary positioning rods. The factors were monitored across the two groups and compared to determine which of the two was superior to the other. Clinical follow-up was done at two weeks and four postoperative to monitor the knee function, as illustrated in table four. Other more acute factors were also accounted for, as shown in Table 2, by monitoring the duration of the procedure and intraoperative bleeding. Extramedullary templates were superior to intramedullary positioning rods in the immediate factors. Still, it had no statistical significance of knee joint function by assessing the angle of deviation and the HSS score. 
Statistical analysis was conducted using IBM's Spss20.0 software. The analysis incorporated various parameters used to compare extramedullary templates and intramedullary positioning rods.

Intraoperative bleeding, operation time, and postoperative drainage were analyzed and compared between the two groups from the acute phase postoperative. The factors defining group similarities like age, body mass index, and hip, knee, and ankle angle deviation were also analyzed. In the follow-up time post-operation, the HSS knee score and the range of knee joint motion, femoral prosthesis flexion, and coronal angle were analyzed. The values of the figures measured were represented as a mean value with a standard deviation $(\bar{x} \pm \mathrm{s})$ with a test level of 0.05 .

\section{Results}

\subsection{General Information about the Surgery}

Table 2 illustrates the safety comparison between the extramedullary and intramedullary groups. The operation time for the extramedullary group was approximately 96.57 minutes with a margin of error of (+/-) 8.576 minutes. In contrast, the intramedullary group had an average time of 104.8 minutes and a margin of error of (+/-) 8.864. Intraoperative bleeding for the extramedullary group was less at approximately $89.97 \mathrm{ml}$, while it was $95.4 \mathrm{ml}$ in the intramedullary group. Postoperative drainage was also more in the intramedullary group as compared to the extramedullary group. The differences between both groups were statistically significant, with a p-value of less than 0.05 . The difference for the operative time was less than 0.001 , intraoperative bleeding had a difference of 0.022 , and postoperative drainage was less than 0.001 .

Table 2

illustrates the safety comparison between the extramedullary and intramedullary groups focusing on operation time, intraoperative blood loss, and postoperative drainage between the extramedullary

localization group and intramedullary localization group from data collected on 35 cases.

\begin{tabular}{|c|c|c|c|}
\hline groups & $\begin{array}{l}\text { operation time } \\
\text { (min) }\end{array}$ & $\begin{array}{l}\text { intraoperative bleeding } \\
(\mathrm{mL})\end{array}$ & $\begin{array}{l}\text { postoperative drainage } \\
(\mathrm{mL})\end{array}$ \\
\hline $\begin{array}{l}\text { Extramedullary localization } \\
\text { group }\end{array}$ & $96.57 \pm 8.576$ & $89.97 \pm 10.051$ & $179.43 \pm 48.443$ \\
\hline $\begin{array}{l}\text { Intramedullary localization } \\
\text { group }\end{array}$ & $104.8 \pm 8.864$ & $95.4 \pm 9.372$ & $229.29 \pm 55.095$ \\
\hline$t$ & -3.947 & -2.337 & -4.021 \\
\hline$P$ & $₫ 0.001$ & 0.022 & $\nabla 0.001$ \\
\hline
\end{tabular}

\subsubsection{Postoperative Prosthesis Installation Angle}


The postoperative HKA deviation in the extramedullary localization group was smaller than in the intramedullary localization group, with a difference of no statistical significance of a p-value more than 0.05. In addition, there was no significant difference in HKA angle deviation, coronal plane angle, and flexion angle of femoral prosthesis $(P>0.05)$, as shown in Table 3 .

Table 3

Extramedullary positioning group and intramedullary positioning group postoperative prosthesis installation angle compared

\begin{tabular}{|llll|}
\hline groups & $\begin{array}{l}\text { HKA angle } \\
\text { deviation }\left(^{\circ}\right)\end{array}$ & $\begin{array}{l}\text { the coronal angle of femoral } \\
\text { prosthesis }\left(^{(}\right)\end{array}$ & $\begin{array}{l}\text { flexion angle of femoral } \\
\text { prosthesis }\left(^{\circ}\right)\end{array}$ \\
\hline $\begin{array}{l}\text { Extramedullary } \\
\text { localization group }\end{array}$ & $2.143 \pm 0.9583$ & $90.334 \pm 2.8565$ & $90.954 \pm 1.7706$ \\
\hline $\begin{array}{l}\text { Intramedullary } \\
\text { localization group }\end{array}$ & $2.046 \pm 1.0947$ & $89.443 \pm 1.8392$ & $90.274 \pm 1.7219$ \\
\hline$t$ & 0.395 & 1.552 & 1.629 \\
\hline$P$ & 0.694 & 0.126 & 0.108 \\
\hline
\end{tabular}

Postoperative knee function

Patient follow-up was scheduled after two weeks then at four weeks postoperative. Table 4 represents the follow-up findings between the two groups at two weeks and four weeks. At four weeks, the HSS score was done, and it is also represented on the table. The differences between the two systems were of no statistical significance based on a p-value of more than 0.05 . Therefore, extramedullary templates were non-superior to intramedullary positioning rods based on knee motion.

Table 4

represents the follow-up findings at two weeks and at four weeks focusing on knee function using the angle of deviation and HSS score between the extramedullary and intramedullary groups.

\begin{tabular}{|llll|}
\hline groups & $\begin{array}{l}\text { 2-week range of motion } \\
\text { of knee joint }\left(^{\circ}\right)\end{array}$ & $\begin{array}{l}\text { 4-week range of motion } \\
\text { of knee joint }\left(^{\circ}\right)\end{array}$ & $\begin{array}{l}\text { 4-week HSS } \\
\text { score (score) }\end{array}$ \\
\hline $\begin{array}{l}\text { Extramedullary } \\
\text { localization group }\end{array}$ & $101.94 \pm 8.321$ & $108.91 \pm 6.28$ & $88.71 \pm 2.456$ \\
\hline $\begin{array}{l}\text { Intramedullary } \\
\text { localization group }\end{array}$ & $101.23 \pm 9.124$ & $108.34 \pm 6.62$ & $88.09 \pm 2.035$ \\
\hline$t$ & 0.342 & 0.371 & 1.166 \\
\hline$P$ & 0.733 & 0.712 & 0.248 \\
\hline
\end{tabular}

\section{Discussion}

The extramedullary positioning osteotomy template is meant to use a self-made femoral extramedullary positioning osteotomy template to perform a distal femoral osteotomy. Therefore, the extramedullary 
avoids most of the risks associated with intramedullary positioning rods. The extramedullary positioning osteotomy template avoids marrow expansion, has significantly less surgical trauma and reduces the risk of intraoperative bleeding and fat embolism [10].In addition, the risk for infection and exposure to radiation is reduced from the reduced preoperative preparation for the procedure. Extramedullary positioning osteotomy templates are particularly significant in presentations where an intramedullary osteotomy positioning rod is contraindicated, such as femoral stem deformations or where the femoral stem is long. It may also find use in contraindications emanating from difficulty aligning the positioning rod and when the anterior arch of the femur is largely resulting from deviations in intramedullary positioning [11]. Therefore, the extramedullary positioning osteotomy template reduces the risks often associated with intramedullary positioning rod insertion.

The template is built on the principle that the force line is perpendicular to the tibia and the femur's osteotomy surface. Upon completion of the standard tibial osteotomy, the distal femoral osteotomy surface may be found in the coronal plane regarding the force line of the lower limb that connects the hip, knee, and the center of the ankle and in the sagittal plane about the anterolateral femoral cortex hence ensuring accuracy while positioning the lower limb force line [12] In addition, $t$ distal femoral osteotomy surface angle may be personalized to avoid a repeat osteotomy due to insufficiency of knee extension clearance [13] That means that preparation for the procedure is less cumbersome than with the intramedullary positioning. The procedure is more predefined than intramedullary rod positioning.

No patient is universal, and that statement is alive with the difference in the valgus angle of the femur that varies with each patient. The self-made extramedullary positioning osteotomy template allows for personalization of the template in line with the valgus angle of the distal femoral osteotomy surface. Intramedullary positioning procedures must incorporate a step to place the space between the tibial and femur on the medial and lateral aspects of the knee joint [14]. The knee must be extended to measure the knee extension gap [15]. Intramedullary positioning often involves re-osteotomy of the distal femur and the proximal tibia due to inadequate osteotomy volume, leading to increased intraoperative time. The increased operation time increased surgical trauma, risk of infection, and increased intraoperative hemorrhage.

The homemade osteotomy templates for extramedullary positioning of the femur allow for personalization of the thickness of the knee extension gap as per the thickness of different producers. Tibial guide thickness is $13 \mathrm{~mm}$ and $14 \mathrm{~mm}$ on each side, respectively. The distal femoral osteotomy template has knee extension gaps set at $17 \mathrm{~mm}, 19 \mathrm{~mm}$, and $21 \mathrm{~mm}$ of the $13 \mathrm{~mm}$ tibial guide side with $18 \mathrm{~mm}, 20 \mathrm{~mm}$, and 22 , on the $14 \mathrm{~mm}$ aspect of the tibial guide plate side. The analysis from the study was a non-superiority trial that showed that the use of extramedullary femoral positioning templates significantly reduced intraoperative time compared to intramedullary positioning [15].In addition, the procedure utilized relatively fewer tools and had reduced surgical trauma with lower risks for infection or operation-related risks. Therefore, the extramedullary plate is superior to the intramedullary rods, as indicated by the reduced risks without any added financial implications. 


\subsection{Accuracy of Femoral Prosthesis Installation}

The analysis of the results revealed that the prosthesis loosened $3 \%$ in those with axial force line shift of $3^{0}$ or lower and $24 \%$ in 8 years post-operation in those with a $3^{0}$ shift or more [16] Deviation after five years in the lower limb force line was $3^{0}$ with an HSS knee score of lower than $3^{0}$ [17]. Application of the extramedullary technique using new mechanical axis marking protocols provides reliable whole limb alignment and is accurate on the standard of the intramedullary technique [18]. The fact that the extramedullary technique uses fewer tools accounts for increased accuracy. Studies have confirmed a hypothesis that instrumentation fails to reach optimal precision in the positioning of tibial components during total knee arthroplasty. With every part of the procedure, instrumentation appeared to increase Varus malalignment and reduce the tibial slope angle[19].

\subsection{Study Limitations}

Of note, all were analyzed except for exposure to radiation and preoperative preparation of the risks associated with intramedullary positioning rods. That is because there were no adjustments in that area between the two groups. The $\mathrm{C}$-arm x-ray was still used to determine the center of the femoral head, and, therefore, the patient was still exposed to considerable amounts of radiation. The process also resulted in the consumption of time before the procedure. Consequently, the indifference in the preparation time and procedure resulted in a lack of necessity to analyze that aspect of the procedure. The study also failed to elicit the impact of the prosthesis on the quality of life of the patients. The prosthesis used was also uniform across both groups, resulting in even more difficulty eliciting accuracy results. The manifestation of the patient's following the procedure may be inaccurate due to similar prostheses for patients with different positioning mechanisms. To resolve the challenges, the study recommended that the $\mathrm{C}$-arm $\mathrm{x}$ ray machine be adjusted to reduce the necessity of prolonged exposure to find the femoral head center. The study also recommended that the functional implications and the implications of the prosthesis on the quality of life be studied in more detail in future studies.

\section{Conclusion}

The study was a non-superiority trial that showed that extramedullary femoral positioning templates significantly reduced intraoperative time compared to intramedullary positioning. Extramedullary positioning osteotomy template avoids marrow expansion, has significantly less surgical trauma and reduces the risk of intraoperative bleeding, fat embolism and infection. Self-made extramedullary positioning osteotomy templates allow for personalization of the template in line with the valgus angle of the distal femoral osteotomy surface. The template demonstrated less surgical trauma hence the lower risk of deformity, less intraoperative bleeding, more safety and less risk for infection than the intramedullary positioning rods. Thus, extramedullary distal femoral osteotomy positioning templates proved superior to intramedullary positioning rods.

\section{Abbreviations}


TKA

total knee arthroplasty

HSS

hospital special surgery

HKA

hp knee ankle

\section{Declarations}

Ethical Approved and Consent to participate: Approved by Department of traumatology, second affiliated hospital of Kunming medical university, and Department of joint surgery, First Affiliated Hospital of Dali University Ethical Committee.

Consent for publication: All authors have reviewed and confirmed the accuracy of the whole manuscript

Consent to participate: obtained

Availability of Data and materials: The data sets used and analyzed during the current study are available from the corresponding author on reasonable request.

Competing Interests: The authors declare that they have no competing interests.

Funding: Not applicable yet

Author contributions:

Substantial contributions to conception and design: $\mathrm{DH}, \mathrm{ZHG}, \mathrm{SZH}, \mathrm{SCHJ}$.

data collection, analysis and interpretation of results: $\mathrm{DH}, \mathrm{SZH}, \mathrm{ZHG}$,

All authors Drafting the article or critically revising it for important intellectual content.

All authors approval of the version to be published.

$\mathrm{DH}, \mathrm{ZHG}, \mathrm{SZH}, \mathrm{SCHJ}$ : Agreement to be accountable for all aspects of the work in ensuring that questions related to the accuracy or integrity of the work are appropriately investigated and resolved.

All authors have read and approved the manuscript.

\section{References}

1. Amro, R. R., Nazarian, D. G., Norris, R. B., Kelly, M. P., \& Booth Jr, R. E. (2001). Suction instrumentation decreases intramedullary pressure, pulmonary embolism during total knee arthroplasty. Univ Penn Orthop J, 14, 55-59. 
2. Bonanzinga, T., Tanzi, P., Neri, M. P., lacono, F., Mazzola, C., Belluati, A., ... \& Marcacci, M. (2018). Evaluation of Blood Loss and Implant Alignment after Total Knee Arthroplasty with Inertial Based Extramedullary Femoral Cutting Guide. Joints, 6(3), 161. doi:10.1055/s-0038-1673404

3. Burnett, R. S. J., \& Barrack, R. L. (2013). Computer-assisted total knee arthroplasty is currently of no proven clinical benefit: a systematic review. Clinical Orthopaedics and Related Research $B, 471(1)$, 264-276. doi:10.1007/s11999-012-2528-8

4. Cheng, T., Zhao, S., Peng, X., \& Zhang, X. (2012). Does computer-assisted surgery improve postoperative leg alignment and implant positioning following total knee arthroplasty? A metaanalysis of randomized controlled trials?. Knee Surgery, Sports Traumatology, Arthroscopy, 20(7), 1307-1322. https://doi.org/10.1007/s00167-011-1588-8

5. Gan, Y., Ding, J., Xu, Y., \& Hou, C. (2015). Accuracy and efficacy of osteotomy in total knee arthroplasty with a patient-specific navigational template. International journal of clinical and experimental medicine, 8(8), 12192.

6. Hoffart, H. E., Langenstein, E., \& Vasak, N. (2012). A prospective study comparing the functional outcome of computer-assisted and conventional total knee replacement. The Journal of bone and joint surgery. British volume, 94(2), 194-199. https://doi.org/10.1302/0301-620X.94B2.27454

7. Hoppe, S., Mainzer, J. D., Frauchiger, L., Ballmer, P. M., Hess, R., \& Zumstein, M. A. (2012). More accurate component alignment in navigated total knee arthroplasty has no clinical benefit at 5-year follow-up. Acta orthopedic, 83(6), 629-633. https://doi.org/10.3109/17453674.2012.747923

8. Hsu, W. H., Hsu, R. W. W., \& Weng, Y. J. (2010). Effect of preoperative deformity on postoperative leg axis in total knee arthroplasty: a prospective randomized study. Knee Surgery, Sports Traumatology, Arthroscopy, 18(10), 1323-1327. https://doi.org/10.1007/s00167-010-1146-9

9. Huang, N. F., Dowsey, M. M., Ee, E., Stoney, J. D., Babazadeh, S., \& Choong, P. F. (2012). Coronal alignment correlates with outcome after total knee arthroplasty: five-year follow-up of a randomized controlled trial. The Journal of arthroplasty, 27(9), 1737-

1741. https://doi.org/10.1016/j.arth.2012.03.058

10. Iorio, R., Bolle, G., Conteduca, F., Valeo, L., Conteduca, J., Mazza, D., \& Ferretti, A. (2013). Accuracy of manual instrumentation of tibial cutting guide in total knee arthroplasty. Knee Surgery, Sports Traumatology, Arthroscopy, 21(10), 2296-2300. https://doi.org/10.1007/s00167-012-2005-7

11. Jämsen, E., Huhtala, H., Puolakka, T., \& Moilanen, T. (2009). Risk factors for infection after knee arthroplasty: a register-based analysis of 43,149 cases. JBJS, 91(1), 38-47. doi:

10.2106/JBJS.G.01686

12. Jeffery, R. S., Morris, R. W., \& Denham, R. A. (1991). Coronal alignment after total knee replacement. The Journal of bone and joint surgery. British volume, 73(5), 709-714. https://doi.org/10.1302/0301620X.73B5.1894655

13. Jeon, S. H., Kim, J. H., Lee, J. M., \& Seo, E. S. (2012). Efficacy of extramedullary femoral component alignment guide system for blood-saving after total knee arthroplasty. Knee surgery \& related research, 24(2), 99. doi: 10.5792/ksrr.2012.24.2.99

Page 13/19 
14. Jung, W. H., Chun, C. W., Lee, J. H., Ha, J. H., \& Jeong, J. H. (2013). The accuracy of the extramedullary and intramedullary femoral alignment system in total knee arthroplasty for varus osteoarthritic knee. Knee Surgery, Sports Traumatology, Arthroscopy, 21(3), 629635. https://doi.org/10.1007/s00167-012-1994-6

15. Lee, C. Y., Huang, T. W., Peng, K. T., Lee, M. S., Hsu, R. W., \& Shen, W. J. (2015). Variability of distal femoral valgus resection angle in patients with end-stage osteoarthritis and genu varum deformity: a radiographic study in an ethnic Asian population. Biomed J, 38(4), 350-355.

16. Matsumoto, K., Mori, N., Ogawa, H., \& Akiyama, H. (2015). Accuracy of a novel extramedullary femoral alignment guide system in primary total knee arthroplasty. Archives of orthopedic and trauma surgery, 135(12), 1743-1748. https://doi.org/10.1007/s00402-015-2318-3

17. Nunley, R. M., Ellison, B. S., Ruh, E. L., Williams, B. M., Foreman, K., Ford, A. D., \& Barrack, R. L. (2012). Are patient-specific cutting blocks cost-effective for total knee arthroplasty?. Clinical Orthopaedics and Related Research $\Theta_{1}$, 470(3), 889-894. doi: 10.1007/s11999-011-2221-3

18. Nunley, R. M., Ellison, B. S., Zhu, J., Ruh, E. L., Howell, S. M., \& Barrack, R. L. (2012). Do patientspecific guides improve coronal alignment in total knee arthroplasty?. Clinical Orthopaedics and Related Research $\Theta, 470(3), 895-902$. doi: 10.1007/s11999-011-2222-2

19. Panjwani, T. R., Mullaji, A., Doshi, K., \& Thakur, H. (2019). Comparison of functional outcomes of computer-assisted vs conventional total knee arthroplasty: a systematic review and meta-analysis of high-quality, prospective studies. The Journal of arthroplasty, 34(3), 586593. https://doi.org/10.1016/j.arth.2018.11.028

20. Roh, Y. W., Kim, T. W., Lee, S., Seong, S. C., \& Lee, M. C. (2013). Is TKA using patient-specific instruments comparable to conventional TKA? A randomized controlled study of one system. Clinical Orthopaedics and Related Research $B, 471(12), 3988-3995$. doi: 10.1007/s11999-013-3206-1

21. Tang, Q., Shang, P., Zheng, G., Xu, H. Z., \& Liu, H. X. (2017). Extramedullary versus intramedullary femoral alignment technique in total knee arthroplasty: a meta-analysis of randomized controlled trials. Journal of orthopedic surgery and research, 12(1), 1-7. https://doi.org/10.1186/s13018-0170582-3

22. van Groningen, B., den Teuling, J. W. A. M., Houterman, S., \& Janssen, R. P. A. (2015). Femoral mechanical-anatomical angle measurements in total knee arthroplasty: analog versus digital. The journal of knee surgery, 28(04), 315-320. DOI: 10.1055/s-0034-1384214

\section{Figures}




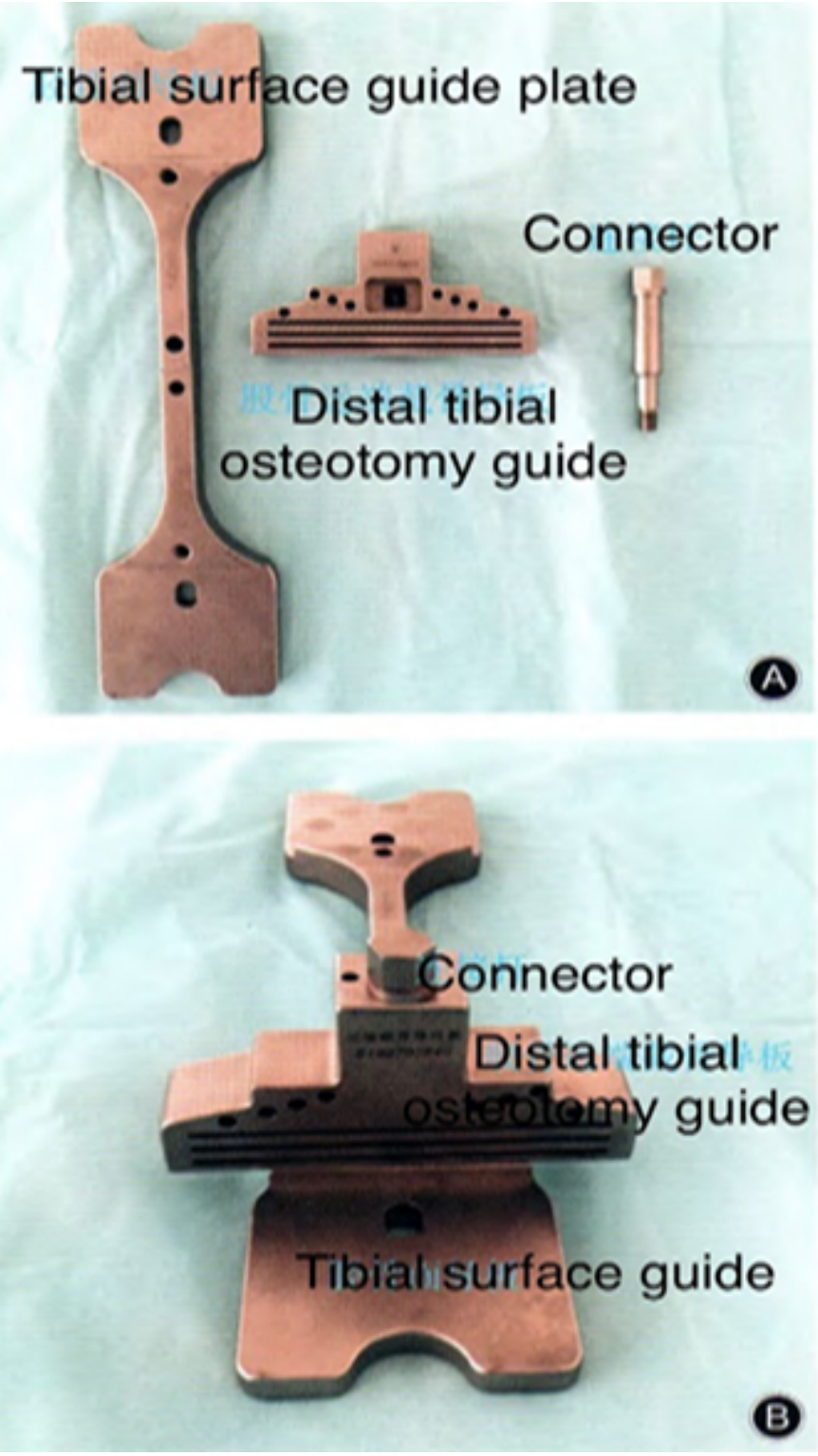

Figure 1

Components of the self-made extramedullary template, patent by Beijing Weigao Yahua Artificial Joint Development Ltd. 

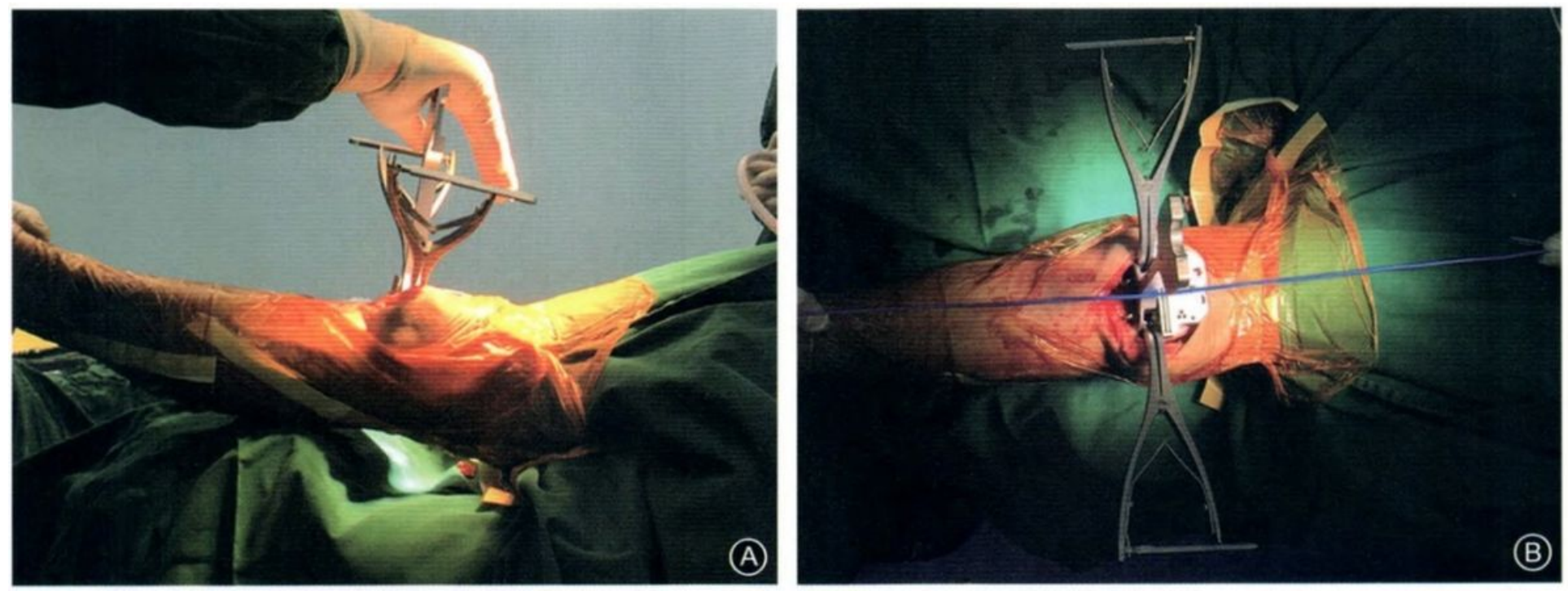

\section{Figure 2}

Spreader clamps were placed in the joint spaces laterally and medially between the proximal tibia and the distal femur. The extramedullary template is then placed in line with the lower limb force line and the distal femur osteotomy hoisted to the distal femur.
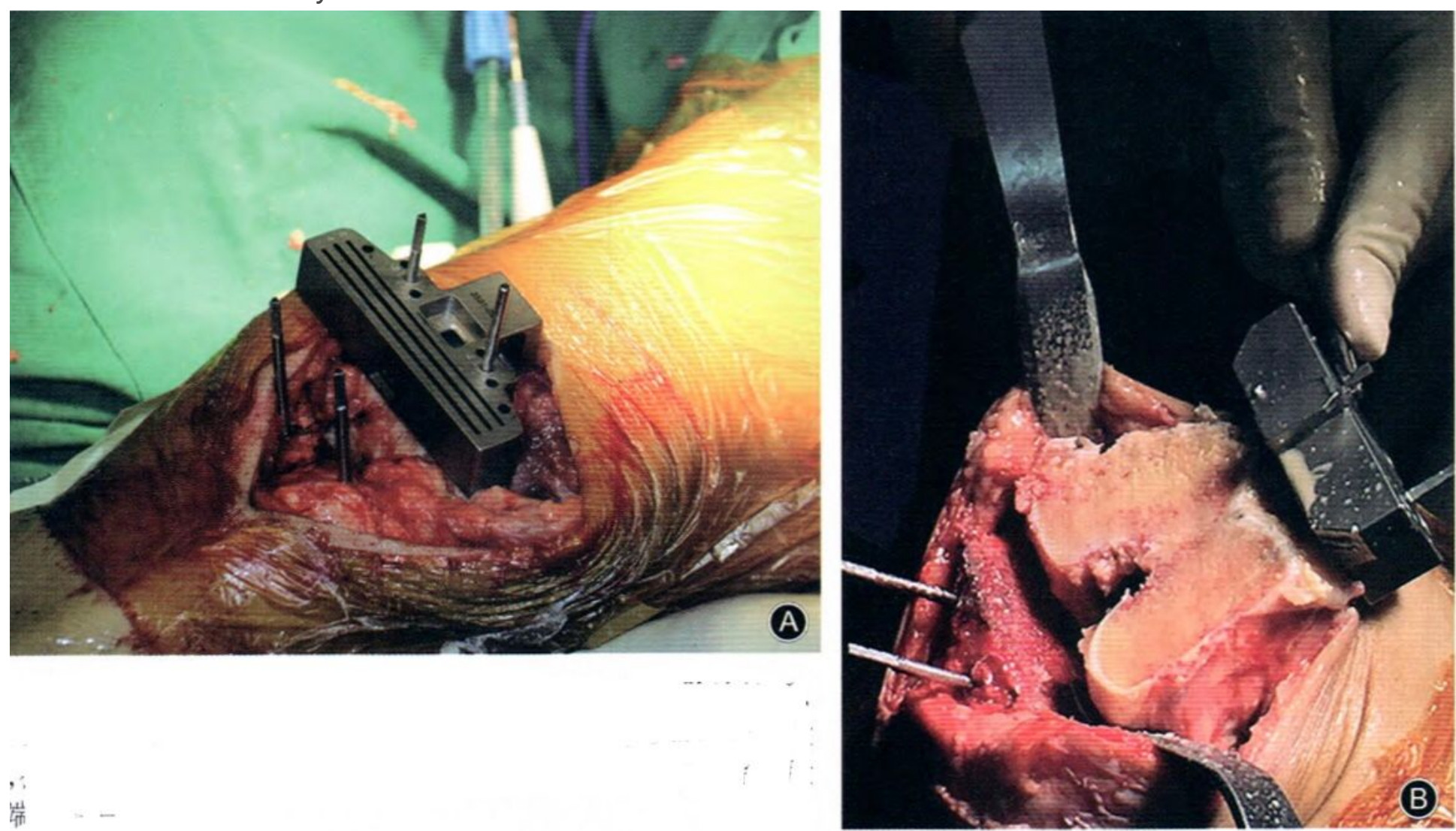

\section{Figure 3}

Distal femur osteotomy guide plate fixed to the distal femur with a fixed screw, remove the connecting rod to separate the tibial surface guide plate from the distal femur osteotomy guide plate, fix the distal femur 
osteotomy guide plate to the side of the femur. Once fixed with fixation screws, the knee is flexed to verify the lower limb force line. Hyperflexion of the knee joint is conformed upon exposure of anterolateral femoral cortex with the osteotomy template.
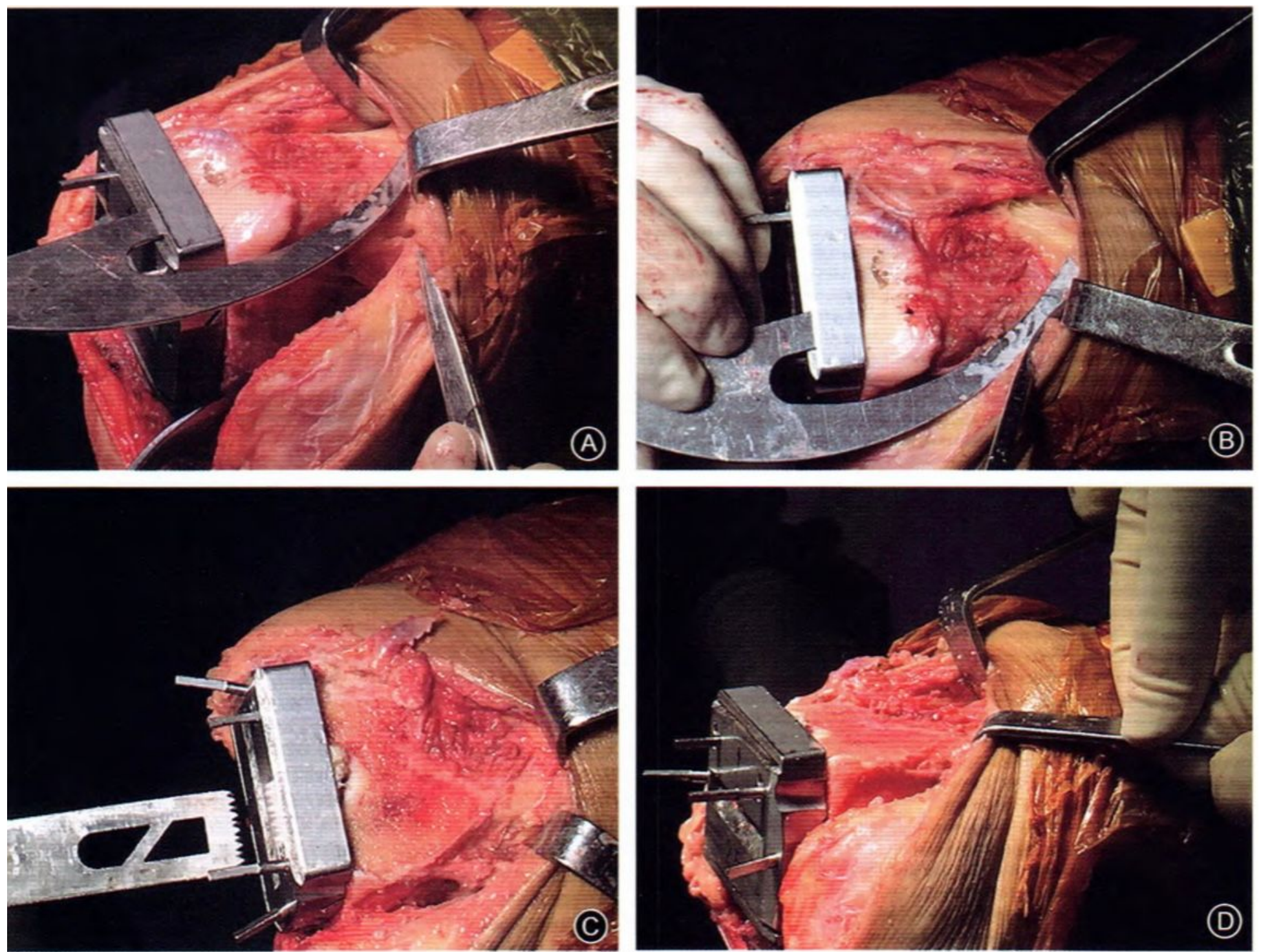

\section{Figure 4}

Verifying flexion or hyperextension on the sagittal plane of the osteotomy line of the anterior condyle of the distal femur After two weeks of the procedure, a frontal and lateral view $\mathrm{x}$-ray was done to assess the HKA knee and coronal angle. 

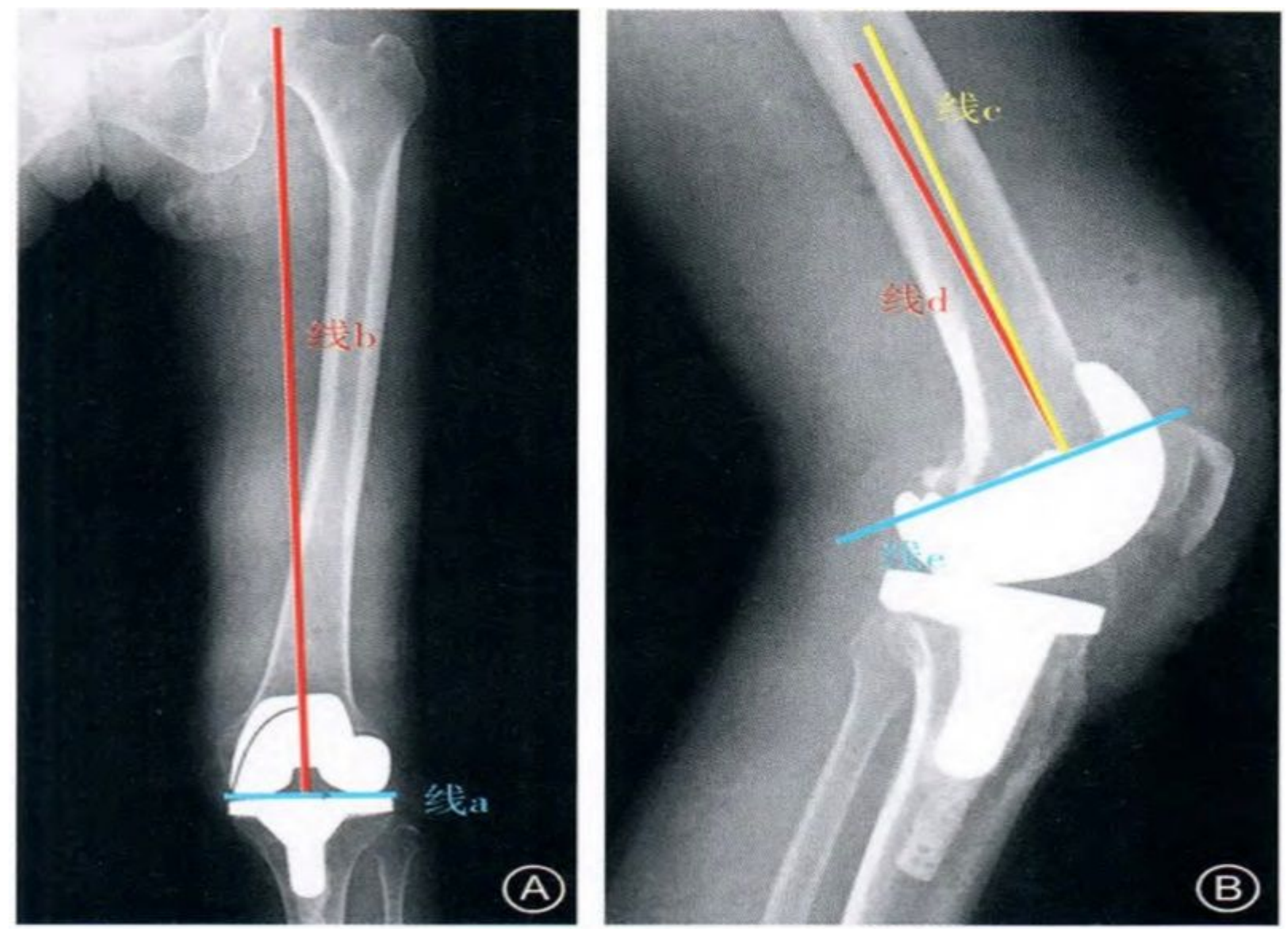

\section{Figure 5}

The coronal angles of A-share, a-stomach and f-segment were measured on the lower limbs' full-length anteroposterior and lateral X-ray films. 

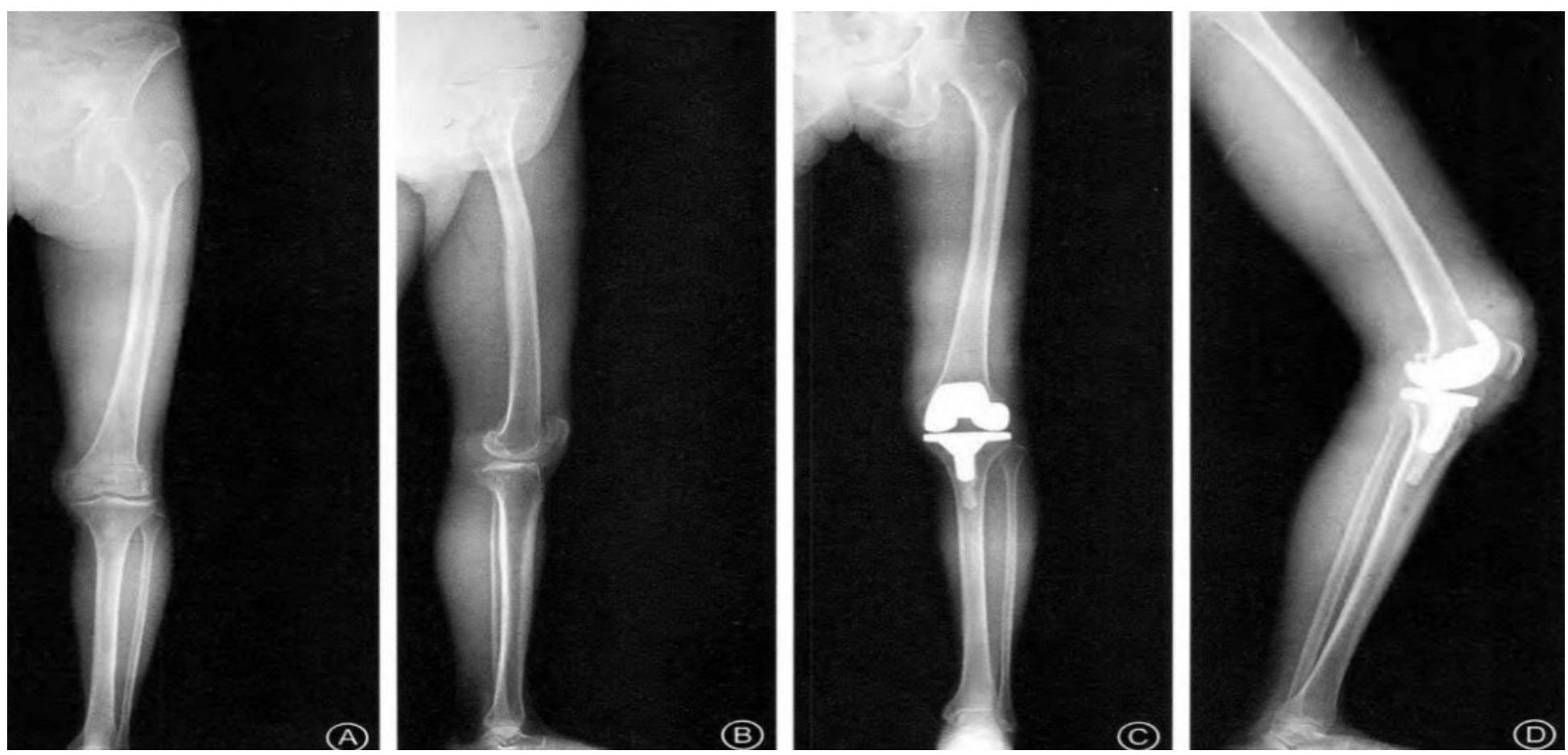

\section{Figure 6}

A 65year old female with osteoarthritis of the left knee underwent total knee arthroplasty. During the operation, the distal femoral osteotomy was performed by the extramedullary localization method. A, B. preoperative full-length anteroposterior and lateral X-ray films of $F$ limb showed HKA angle deviation of 8.4. The HKA angle was 189凶 Angle deviation 1.), The coronal angle of the femoral prosthesis was 89.6. The lateral X-ray film of D's lower limb showed that the flexion angle of the femoral prosthesis was 1.2. 